\title{
ACTITUDES HACIA LA SEXUALIDAD Y BIENESTAR PSICOLÓGICO EN PERSONAS MAYORES
}

\author{
Vicente Morell-Mengual \\ Dpto. de Psicología Evolutiva y de la Educación, Universitat de Valènci \\ vicente.morell@uv.es \\ Roberta Ceccato \\ Dpto. de Psicología Evolutiva y de la Educación, \\ Universitat de València \\ Juan Enrique Nebot-García \\ Dpto. de Psicología Básica, Clínica y Psicobiología, \\ Universitat Jaume I \\ Isabel Chaves \\ Dpto. de Psicología Evolutiva y de la Educación, \\ Universitat de València \\ María Dolores Gil- Llario \\ Dpto. de Psicología Evolutiva y de la Educación, \\ Universitat de València
}

Fecha de Recepción: 2 Marzo 2018

Fecha de Admisión: 10 Abril 2018

\section{RESUMEN}

El estudio de los factores que influyen en el proceso de envejecimiento activo y saludable constituye uno de los principales intereses de la psicogerontología. En este sentido, el bienestar psicológico es un indicador fiable que asegura el éxito de dicho proceso, mientras que la sexualidad se convierte en una variable moduladora relevante. El objetivo de este trabajo es determinar si las actitudes hacia la sexualidad y el bienestar psicológico de las personas mayores están moduladas por el tipo de residencia, el estado civil y el grado de autonomía. Para ello se utiliza la Escala de Actitudes sobre la Sexualidad en la Vejez y la Escala de Bienestar Psicológico en una muestra de 70 personas, 35 mujeres y 35 hombres, con edades comprendidas entre los 65 y los 95 años ( $M=76.78$; DT = 8.3). Los resultados revelan que, en general, las actitudes más liberales hacia la sexualidad y el mayor grado de bienestar psicológico se asocian significativamente con el hecho de vivir fuera de las instituciones residenciales y mantener una relación sentimental con una pareja estable. Por su parte, el grado de autonomía solo alcanza significación estadística en el bienestar psicológico, pero no en las actitudes hacia la sexualidad. Puesto que las personas mayores representan un sector de la población estigmatizado (con numerosos mitos y creencias negativas asociadas), este estudio contribuye a crear una concepción más positiva del proceso de envejecimiento, normalizando los 


\section{ACTITUDES HACIA LA SEXUALIDAD Y BIENESTAR PSICOLÓGICO EN PERSONAS MAYORES}

comportamientos y enfatizando las variables a considerar en la elaboración de programas de optimización evolutiva.

Palabras clave: actitudes; sexualidad; bienestar psicológico; personas mayores

\section{ABSTRACT}

Attitudes toward sexuality and psychological well-being in older people.

The study of the factors that influence the active and healthy aging process is one of the main interests of psychogerontology. In this sense, psychological well-being is a reliable indicator that ensures the success of this process, while sexuality becomes a relevant modulating variable. The aim of this paper is to determine if attitudes towards sexuality and psychological well-being of ederly people are modulated by residence type, marital status and degree of autonomy. For this purpose, Ageing Attitude Scale on Sexuality and Psychological Well-Being Scale is used in a sample of 70 people, 35 women and 35 men, from 65 to 95 years old $(M=76.78$; $D T=8.3)$. The results show that, in general, more liberal attitudes towards sexuality and greater psychological well-being are significantly associated with living outside residential institutions and maintaining a sentimental relationship with a stable partner. On the other hand, the degree of autonomy only obtains statistical significance in psychological well-being but not in attitudes towards sexuality. In conclusion, ederly people represent a stigmatised segment of the population (with many associated myths and negative beliefs), for this reason, this study contributes to create a positive conception of the aging process, normalizing behaviors and emphasizing the variables to be considered in the elaboration of intervention programs.

Keywords: attitudes; sexuality; psychological well-being; elderly people

\section{INTRODUCCIÓN}

La concepción clásica de la psicogerontología se centra en el estudio de los déficits y características patológicas asociadas a la edad. No obstante, el progresivo incremento de la esperanza de vida unido al surgimiento de la psicología positiva ha propiciado un cambio en el foco de interés hacia aspectos más positivos tales como, la actividad, el optimismo, la vitalidad y la productividad (Fernández-Ballesteros, 2009); entendiendo el envejecimiento como un proceso que implica al mismo tiempo ganancias y pérdidas (Baltes, 1987; Heckhausen y Schulz, 1993). Este nuevo paradigma está centrado en el envejecimiento con éxito y se caracteriza por el análisis de los criterios y las condiciones que permiten mantener un adecuado funcionamiento físico, mental y social. El envejecimiento exitoso es un concepto relativamente nuevo compuesto por un amplio conjunto de factores biopsicosociales que contemplan, entre otros aspectos, una baja probabilidad de enfermedad y discapacidad; un alto grado de funcionamiento físico y mental; y un alto grado de participación social (Fernández-Ballesteros, 2009; Fernández-Ballesteros et al., 2010).

En la actualidad, existen numerosos enfoques y teorías que intentan abordar el envejecimiento con éxito y diversas investigaciones que intentan conceptualizar los componentes para que este proceso se lleve a cabo. En este sentido, la perspectiva eudaimónica considera el bienestar psicológico como un indicador de funcionamiento positivo que posibilita el desarrollo de las capacidades personales. Esta perspectiva ha sido sistematizada por Ryff y Singer (2008) en su modelo multidimensional del bienestar psicológico compuesto por seis dimensiones: autoaceptación, crecimiento personal, propósito de vida, relaciones positivas con otros, dominio ambiental y autonomía.

El bienestar subjetivo es considerado un concepto psicológico relativamente semejante a la satisfacción con la vida (Lyubomirsky, King y Diener, 2005) que está influido por diferentes características sociodemográficas como el sexo y el estado civil (Vivaldi y Barra, 2012) y el grado de institucionalización (Molina, Meléndez y Navarro, 2008). Diversos estudios determinan que las perso- 
nas que viven en pareja, ya sea noviazgo o matrimonio, muestran un mayor grado de bienestar (Vivaldi y Barra, 2012). Según Pinguart y Sorense (2001) las personas divorciadas, solteras o viudas pueden ser más propensas a enfermar y presentar mayores índices de mortalidad y accidentes que las personas casadas, debido probablemente a la falta de apoyo social percibido.

Otro de los factores que puede ocasionar una disminución de la interacción social en las personas mayores es su institucionalización en centros residenciales. El ingreso en este tipo de centros es uno de los acontecimientos que mayores repercusiones provoca en las relaciones sociales, ya que la institucionalización puede incrementar los contactos sociales o provocar un mayor aislamiento (Fernández Ballesteros, 2002; 2009). En general, las personas mayores que viven en sus domicilios cuentan con más recursos adaptativos que las personas institucionalizadas (Feldberg y Stefani, 2006). Además, el aspecto relacional es superior en personas no institucionalizados las cuales además alcanzan mayores porcentajes de bienestar psicológico relacionado con el apoyo social familiar (Molina et al., 2008).

La necesidad de interacción, intimidad y afecto de una persona no terminan en la vejez, es más, el interés por la sexualidad y la actividad sexual es un indicador de calidad de vida en el adulto mayor (Guadarrama, Ortiz, Moreno y Gonzales, 2010). El desarrollo sexual humano es un proceso que abarca toda la vida, siendo una dimensión que solo desaparece con la muerte. La sexualidad es una característica que acompaña al ser humano desde que nace y que está íntimamente relacionada con la búsqueda de afecto, ternura, contacto, placer e intimidad; en ella influyen nuestros pensamientos, la percepción que tenemos de las relaciones interpersonales y la salud (Orihuela, Gómez y Fumero, 2001; Taylor y Gosney, 2011). En la actualidad numerosos estudios confirman la influencia que ejerce el hecho de tener una sexualidad satisfactoria sobre la calidad de vida de los adultos mayores (Guadarrama et al., 2010).

A pesar de las múltiples aportaciones que la sexualidad brinda al ser humano todavía existe una visión negativa donde la sociedad encasilla al adulto mayor como carente de sexualidad. Los estereotipos negativos acerca de la sexualidad en la vejez afirman que las personas mayores no tienen deseos ni necesidades sexuales, que el sexo en esta etapa de la vida es prescindible y hasta que las manifestaciones sexuales en esta etapa son patológicas (Freixas, Luque y Reina, 2010; Rheaume y Mitty, 2008). Esta actitud conservadora no es exclusiva de jóvenes 0 adultos, sino que las propias personas mayores también tienden a asumirlas, modificando y suprimiendo sus propios deseos por considerarlos inapropiadas (Cerquera, Galvis y Cala, 2012; Cerquera, López, Núñez y Porras, 2013). Además, algunas personas mayores con intenso deseo sexual experimentan, por desconocimiento o presión social, un sentimiento de culpabilidad y vergüenza (Guadarrama et al., 2010). Los factores implicados en los cambios de comportamiento sexual del adulto mayor son, por un lado, el grado de autonomía, entendiendo ésta como las modificaciones funcionales en las distintas etapas del ciclo sexual que puede verse alterada y, por otro lado, el estado relacional, siendo la viudez uno de los factores más importantes (Cerquera, et al., 2012; Rheaume y Mitty, 2008).

En resumen, estos resultados revelan el desconocimiento que existe sobre la influencia que ejercen algunos factores psicosociales sobre la sexualidad y la calidad de vida de las personas mayores. Por tanto, el objetivo de este trabajo es determinar si las actitudes hacia la sexualidad y el bienestar psicológico de las personas mayores están moduladas por el tipo de residencia, el estado civil y el grado de autonomía.

\section{MÉTODO}

\section{Participantes}

La muestra está compuesta por 70 personas, 35 hombres (54.3\%) y 35 mujeres (45.7\%), con 


\section{ACTITUDES HACIA LA SEXUALIDAD Y BIENESTAR PSICOLÓGICO EN PERSONAS MAYORES}

edades comprendidas entre los 65 y los 95 años ( $M=76.78$; DT = 8.30). Según el tipo de residencia, el $51 \%$ viven en contexto familiar y el $49 \%$ en contexto residencial. El $47.1 \%$ tiene pareja estable y el $54.3 \%$ es totalmente autónomo frente al $45.7 \%$ que tiene un grado de autonomía media.

\section{Instrumentos}

Escala de Bienestar Psicológico (Díaz et al., 2004; Ryff, 1989). Instrumento compuesto por 39 ítems que evalúa seis dimensiones del modelo multidimensional de bienestar psicológico: autoaceptación, relaciones positivas con otras personas, autonomía, dominio del entorno, propósito en la vida, y crecimiento personal. Se contesta mediante una escala tipo Likert con cuatro opciones de respuesta que van desde 1 «muy en desacuerdo» a 4 «muy de acuerdo». El Alpha de Cronbach obtenido en este estudio es de .76

Escala de Actitudes sobre la Sexualidad en la Vejez (López, 2012). Instrumento compuesto por 39 ítems que evalúa dos tipos de actitudes hacia la sexualidad de las personas mayores: (1) actitud liberal (por ejemplo: "Me gustaría saber más acerca de los cambios en el funcionamiento sexual de las personas mayores") y (2) actitud conservadora (por ejemplo: "Una persona de más de 65 años que manifiesta interés sexual se deshonra así mismo"). Se contesta mediante una escala tipo Likert con cuatro opciones de respuesta que van desde 1 «muy en desacuerdo» a 4 «muy de acuerdo». La consistencia interna original del cuestionario es de .85. En la presente investigación se obtiene una alfa de Cronbach de .82

\section{Procedimiento}

Para acceder a la población que no vive en contexto residencial se contactó con diversos centros municipales para la tercera edad. En primer lugar, se solicitó una reunión con el presidente de la entidad para explicarle las características y los objetivos del estudio. Posteriormente, se realizaron las sesiones de evaluación con aquellas personas que manifestaron su interés en participar.

Para acceder a las personas que viven en contexto residencial se contactó con tres centros residenciales solicitándoles su participación en esta investigación. Tras la reunión con la dirección del centro, se contactó con los residentes para solicitarles su participación. Con las personas dispuestas a colaborar se realizó una sesión de evaluación en la que se administraron los cuestionarios mediante una entrevista semiestructurada.

En ambos casos, las sesiones de evaluación se realizaron en un espacio cedido por los centros, preservando en todo momento la intimidad de los residentes. La duración osciló entre 30 y 60 minutos.

\section{Análisis de datos}

Se realizan análisis comparativos con las dos variables dependientes en función del tipo de residencia, el estado civil y el grado de autonomía mediante pruebas t. Se utiliza el programa estadístico IBM SPSS Statistics 24.

\section{RESULTADOS}

Los análisis estadísticos realizados en función del tipo de residencia (ver tabla 1), revelan que las puntuaciones obtenidas por el grupo de personas que no viven en una institución son estadísticamente superiores, en todas las variables exploradas, en comparación con las de las personas que viven en contexto residencial. Este hallazgo indica que el bienestar psicológico y la actitud liberal hacia la sexualidad es superior en aquellas personas que no viven en una residencia. La actitud conservadora hacia la sexualidad es la única variable cuya puntuación media es estadísticamente superior en el grupo de personas que viven en contexto residencial. 
Por lo que se refiere a la relación de pareja (ver tabla 2), las puntuaciones medias de quienes viven en pareja son significativamente superiores a las de quienes viven sin pareja en las variables: relaciones positivas ( $M=19.09$ vs. $M=16.01 ; p=.01$ ), autonomía $(M=19.21$ vs. $16.95 ; p=.033$ ), dominio del entorno ( $M=25.52$ vs. $M=22.38 ; p=.05$ ) y actitud liberal hacia la sexualidad $(M=$ 45.30 vs. $M=37.30 ; p=.009$ ). Por su parte, las personas que viven sin pareja muestran una actitud más conservadora hacia la sexualidad, si bien las diferencias entre ambos grupos no alcanzan significación estadística.

Tabla 1.

Análisis comparativo en función del tipo de residencia

\begin{tabular}{|c|c|c|c|c|c|}
\hline & \multicolumn{2}{|c|}{ Residencia } & \multicolumn{2}{|c|}{ No residencia } & \multirow{2}{*}{$\mathrm{t}$} \\
\hline & M & DT & M & DT & \\
\hline Autoaceptación & 15.23 & 4.92 & 19.57 & 3.79 & $-4.135^{* *}$ \\
\hline Crecimiento personal & 16.80 & 5.38 & 22.01 & 4.37 & $-4.435^{* *}$ \\
\hline Propósito en la vida & 16.14 & 4.89 & 20.14 & 3.95 & $-3.693^{* *}$ \\
\hline Relaciones positivas & 14.78 & 4.99 & 20.14 & 3.46 & $-5.236^{* *}$ \\
\hline Autonomía & 16.26 & 4.61 & 19.77 & 3.57 & $-3.567^{* *}$ \\
\hline Dominio del entorno & 19.82 & 5.89 & 27.89 & 5.23 & $-6.053^{* *}$ \\
\hline Actitud liberal (sexualidad) & 34.57 & 13.75 & 47.54 & 7.90 & $-4.850^{* *}$ \\
\hline Actitud conservadora (sexualidad) & 62.94 & 21.09 & 42.46 & 15.66 & $4.614^{* *}$ \\
\hline
\end{tabular}

Nota: ${ }^{* *} \mathrm{p}<.01$

Tabla 2.

Análisis comparativo en función de la situación de pareja

\begin{tabular}{|c|c|c|c|c|c|}
\hline & \multicolumn{2}{|c|}{ Con pareja } & \multicolumn{2}{|c|}{ Sin pareja } & \multirow{2}{*}{$\mathrm{t}$} \\
\hline & M & DT & M & DT & \\
\hline Autoaceptación & 18.56 & 5.32 & 16.38 & 4.26 & 1.889 \\
\hline Crecimiento personal & 20.56 & 6.24 & 18.38 & 4.66 & 1.658 \\
\hline Propósito en la vida & 18.85 & 4.99 & 17.49 & 4.78 & 1.166 \\
\hline Relaciones positivas & 19.09 & 4.73 & 16.01 & 4.93 & $2.669^{* *}$ \\
\hline Autonomía & 19.21 & 4.36 & 16.95 & 4.32 & $2.188^{*}$ \\
\hline Dominio del entorno & 25.52 & 7.24 & 22.38 & 6.22 & $1.949^{*}$ \\
\hline Actitud liberal (sexualidad) & 45.30 & 10.94 & 37.30 & 13.50 & $2.705^{* *}$ \\
\hline Actitud conservadora (sexualidad) & 48.24 & 20.60 & 56.68 & 21.07 & -1.689 \\
\hline
\end{tabular}

Nota: ${ }^{*} \mathrm{p}<.05 ;{ }^{* *} \mathrm{p}<.01$

Con respecto al grado de autonomía (ver tabla 3) observamos que las personas con mayor grado de autonomía muestran puntuaciones significativamente superiores en crecimiento personal ( $M=20.79$ vs. $17.75 ; p=.021$ ) y en propósito de vida ( $M=19.53$ vs. $M=16.47 ; p=.009)$ con respecto a las puntuaciones que obtienen las personas con menor grado de autonomía. Por lo que se refiere a la actitud hacia la sexualidad observamos, congruentemente con los resultados anteriores, 


\section{ACTITUDES HACIA LA SEXUALIDAD Y BIENESTAR PSICOLÓGICO EN PERSONAS MAYORES}

que las personas con mayor autonomía muestran una actitud más liberal hacia la sexualidad $(M=$ 41.73 vs. $M=40.31$ ) mientras que las personas con menor autonomía muestran actitudes más conservadoras ( $M=51.68$ vs. $M=53.91)$, no obstante, ninguna de las comparaciones alcanza significación estadística.

Tabla 3.

Análisis comparativo en función del grado de autonomía

\begin{tabular}{|c|c|c|c|c|c|}
\hline & \multicolumn{2}{|c|}{ Autonomía total } & \multicolumn{2}{|c|}{ Autonomía media } & \multirow{2}{*}{$\mathrm{t}$} \\
\hline & $\mathrm{M}$ & DT & M & DT & \\
\hline Autoaceptación & 17.92 & 4.78 & 16.78 & 5.01 & .973 \\
\hline Crecimiento personal & 20.79 & 5.50 & 17.75 & 5.1 & $2.367^{*}$ \\
\hline Propósito en la vida & 19.53 & 4.36 & 16.47 & 5.034 & $2.723^{* *}$ \\
\hline Relaciones positivas & 17.53 & 5.45 & 17.38 & 4.61 & .124 \\
\hline Autonomía & 18.02 & 4.30 & 17.06 & 4.51 & 1.660 \\
\hline Dominio del entorno & 24.71 & 7.28 & 22.84 & 6.27 & 1.137 \\
\hline Actitud liberal (sexualidad) & 41.72 & 13.60 & 40.31 & 12.22 & .443 \\
\hline Actitud conservadora (sexualidad) & 51.68 & 20.83 & 53.91 & 20.83 & -.043 \\
\hline
\end{tabular}

Nota: ${ }^{*} \mathrm{p}<.05 ;{ }^{* *} \mathrm{p}<.01$

\section{DISCUSIÓN}

El objetivo de nuestra investigación era determinar si las actitudes hacia la sexualidad y el bienestar psicológico de las personas mayores están moduladas por el tipo de residencia, el estado civil y el grado de autonomía.

Los resultados indican que las personas que viven en contexto residencial se caracterizan por una actitud más conservadora hacia la sexualidad mostrando además un menor bienestar psicológico, causado probablemente por la necesidad de adaptarse a un nuevo sistema con la merma en la capacidad de decisión que en muchos casos conlleva. Estos resultados coinciden con los obtenidos por Fernández-Ballesteros (2009) quien concluye que las relaciones interpersonales favorecen el desarrollo social y psicológico y que estas relaciones pueden verse negativamente influenciadas al vivir en una institución residencial.

El ingreso en una residencia es uno de los acontecimientos que mayor influencia ejerce sobre las relaciones sociales (Fernández-Ballester, 2009), que son de peor calidad en las personas que viven en contextos residenciales, lo cual es percibido incluso por el entorno familiar (Molina y cols., 2008). En esta línea, Stefani y Feldberg (2006) encuentran que las personas mayores que viven en sus domicilios cuentan con más recursos adaptativos que las personas institucionalizadas.

Por lo que se refiere a la relación de pareja, nuestros resultados indican que las personas que viven en pareja muestran actitudes más liberales hacia la sexualidad ya que no manifiestan problemas para expresar sus opiniones ni suelen estar influenciados por las opiniones de los demás; suelen tener confianza en sus ideales y convicciones; muestran un amplio abanico de relaciones cercanas y de confianza; poseen una mejor concepción de sus propias vidas; y disfrutan haciendo planes de futuro. En este sentido, la actitud conservadora hacia la sexualidad podría ser una barrera para ampliar las relaciones sociales, íntimas y cercanas lo que, a su vez, repercutiría en un empeoramiento de la calidad de vida.

Un estudio realizado por Vivaldi y Barra (2012) encontró que las personas mayores con pareja 
informan de mayores niveles de bienestar psicológico en comparación con aquellos que no la tienen, siendo este aspecto un factor relevante para el bienestar psicológico. Congruentemente, Feldberg y Stefani (2006) argumentan que los factores sociales, relacionales y sentimentales son de vital importancia en la tercera edad encontrando que tras la muerte de su cónyuge muchas mujeres dejan de tener relaciones sexuales.

Los prejuicios que la sociedad tienen acerca de la sexualidad en la tercera edad son, en gran parte, los responsables de la soledad de las personas que han perdido a sus parejas (Pinquart y Sorensen, 2001). Según Rheaume y Mitty (2008) aunque la sexualidad es una energía vital que acompaña al ser humano desde que nace hasta que muere, existen factores sociales como el matrimonio que pueden influir en su expresión. La sexualidad es un elemento vital importante de las personas mayores al moderar los efectos negativos del estrés y contribuir así al bienestar y la satisfacción con la vida (Freixas et al., 2010; Guadarrama et al., 2010; Taylor y Gosney, 2011).

Los resultados obtenidos en relación al grado de autonomía determinan que las personas con un buen nivel de autonomía muestran estilos de vida saludables; creen que es importante tener nuevas experiencias y disfrutan haciendo planes. Por tanto, la percepción de una buena salud física influye positivamente en las expectativas vitales y en los planes de futuro. Satorres (2013) afirma que lo más importante no es añadir años a la vida, sino vida a los años. El envejecimiento también puede ser una etapa que facilita el desarrollo de entornos favorables, con mayores oportunidades de participación e interacción social (Feldberg y Stefani, 2006).

A la vista de los resultados obtenidos, podemos afirmar la influencia que ejercen algunas variables psicosociales sobre el bienestar psicológico y las actitudes hacia la sexualidad de las personas mayores. Siendo la vejez un sector de la población estigmatizado con creencias y mitos negativos, estudios como el que presentamos contribuyen a crear una concepción más positiva del envejecimiento, normalizando sus comportamientos y ampliando el conocimiento para trabajar en su desarrollo. Por tanto, estos resultados deben tenerse en cuenta en la elaboración de programas psicoeducativos dirigidos a mejorar las actitudes hacia la sexualidad de las personas mayores.

\section{REFERENCIAS}

Baltes, P. B. (1987). Theoretical propositions of life span developmental psychology: on the dynamics of growth and decline. Developmental Psychology, 23(5), 611-626.

Cerquera, A., López, K., Núñez, Y., \& Porras, E. (2013). Sexualidad femenina en la tercera edad. Informes Psicológicos, 13(2), 135-147.

Cerquera, A.M., Galvis, M.J. y Cala, M.L. (2012). Amor, sexualidad e inicio de nuevas relaciones en la vejez: percepción de tres grupos etarios. Psychologia, 6(2), 73-81.

Díaz, D., Rodríguez-Carvajal, R., Blanco, A., Moreno-Jiménez, B., Gallardo, I., ... Van Dierendonck, D. (2006). Adaptación española de las escalas de bienestar psicológico de Ryff. Psicothema, 18(3), 572-577.

Fernández-Ballesteros, R. (2002). Vivir con vitalidad. Madrid: Pirámide

Fernández-Ballesteros, R. (2009). Envejecimiento activo: contribuciones de la psicología. Madrid: Pirámide.

Fernández-Ballesteros, R., Zamarrón, M.D., López, M.D., Molina, M.A., Díez, J., Montero, P., ... Schettini, R. (2010). Envejecimiento con éxito: criterios y predictores. Psicothema, 22(4), 641647.

Freixas, A., Luque, B. y Reina, A. (2010). Secretos y silencios en torno a la sexualidad de las mujeres mayores. Debate Feminista, 42, 35-51

Guadarrama, R., Ortiz, M., Moreno, Y. y Gonzales, A. (2010). Características de la actividad sexual 


\section{ACTITUDES HACIA LA SEXUALIDAD Y BIENESTAR PSICOLÓGICO EN PERSONAS MAYORES}

de los adultos mayores y su relación con su calidad de vida. Revista de Especialidades MédicoQuirúrgicas, 15(2), 72-79.

Heckhausen, J. y Schulz, R. (1993). Optimization by selection and compensation: Balancing primary and secondary control in life span development. International Journal of Behavioral Development, 16(2), 287-303.

López, F. (2012). Sexualidad y afectos en la vejez. Madrid: Pirámide.

Lyubomirsky, S., King, L. y Diener, E. (2005). The Benefits of Frequent Positive Affect: Does Happiness lead to Success? Psychological Bulletin, 131(6), 803-855.

Molina, C., Meléndez, J.C. y Navarro, E. (2008). Bienestar y calidad de vida en ancianos institucionalizados y no institucionalizados. Anales de Psicología, 24(2), 312-319.

Orihuela, J., Gómez, M. y Fumero, M. (2001). Sexualidad en el anciano: un elemento importante en su calidad de vida. Revista Cubana de Medicina General Integral, 17(6), 545-547.

Pinquart, M. y Sorensen, S. (2001). Influences on loneliness in older adults: A meta-analysis. Basic and applied social psychology, 23(4), 245-266.

Rheaume, C. y Mitty, E. (2008). Sexuality and Intimacy in Older Adults. Geriatric Nursing, 29(5), 342-349.

Ryff, C. (1989). Beyond Ponce de Leon and life satisfaction: New directions in quest of successful aging. International Journal of Behavioral Development, 12(1), 35-55.

Ryff, C. D. y Singer, B. H. (2008). Know thyself and become what you are: A eudaimonic approach to psychological well-being. Journal of Happiness Studies, 9(1), 13-39.

Satorres, E. (2013). Bienestar psicológico en la vejez y su relación con la capacidad funcional y la satisfacción vital (tesis doctoral). Universitat de València, Valencia.

Stefani, D. y Feldberg, C. (2006). Estrés y estilos de afrontamiento en la vejez: Un estudio comparativo en senescentes argentinos institucionalizados y no institucionalizados. Anales de Psicología, 22(2), 267-272.

Taylor, A. y Gosney, M.A. (2011). Sexuality in older age: essential considerations for healthcare professionals. Age and Ageing, 40(5), 538-543.

Vivaldi, F. y Barra, E. (2012). Bienestar psicológico, apoyo social percibido y percepción de salud en adultos mayores. Terapia psicológica, 30(2), 23-29. 